TAIWANESE JOURNAL OF MATHEMATICS

Vol. 14, No. 4, pp. 1571-1576, August 2010

This paper is available online at http://www.tjm.nsysu.edu.tw/

\title{
A MASCHKE-TYPE THEOREM FOR PARTIAL ENTWINING STRUCTURE
}

Ling Jia

\begin{abstract}
In this paper we first prove that the forgetful functor from the category of partial entwined modules to that of modules has a right adjoint functor. Then we give a Maschke-type theorem for a partial entwined module.
\end{abstract}

\section{INTRODUCTION}

During the study of operator algebras partial actions of groups have been introduced by Exel [8]. With the further development many positive results have been proposed [5],[6],[7]. S.Caenepeel and the other authors developed a theory of partial actions of Hopf algebras [3] and pointed that its Galois theory have a remarkable analogy with that of a weak Hopf algebra [4].

In this paper, we first recall definitions of partial entwining structures and give some examples. Then we prove that the forgetful functor from the category of partial entwined modules to the category of modules has a right adjoint functor. Finally we give a Maschke-type theorem of a partial entwined module which generalizes the relevant results of Doi-Hopf modules and entwined modules [1, 2].

\section{Conventions.}

Throughout the paper $k$ is a field. We use the standard Sweedler's notation for comultiplication $\Delta$ and comodule. The identity map from any $k$-space $V$ to itself is denoted by $i d_{V}$.

Definition 1. Let $A$ be a k-algebra, $C$ a k-coalgebra, and $\psi: C \otimes A \rightarrow A \otimes C$ be a k-map. The triple $(A, C, \psi)$ is called a right-right partial entwining structure if the following are satisfied for any $a, b \in A, c \in C$ :

Received December 11, 2007, accepted December 13, 2008.

Communicated by Ruibin Zhang.

2000 Mathematics Subject Classification: 16D80, 16D99.

Key words and phrases: Partial entwining structure, Maschke theorem.

Project supported by the Funds for the Doctors of Ludong University, LY20062703. 
(1) $\sum(a b)_{\psi} \otimes c^{\psi}=\sum a_{\psi} b_{\phi} \otimes c^{\psi \phi}$;

(2) $\sum a_{\psi} 1_{\phi} \otimes c^{\psi}{ }_{1}^{\phi} \otimes c^{\psi}{ }_{2}=\sum a_{\psi \phi} \otimes c_{1}{ }^{\phi} \otimes c_{2}{ }^{\psi}$;

(3) $\sum \varepsilon\left(c^{\psi}\right) a_{\psi}=\varepsilon(c) a$.

A left-left partial entwining structure is a triple $(A, C, \psi)$, where $A$ is a $k$ algebra, $C$ a $k$-coalgebra, and $\psi: C \otimes A \rightarrow A \otimes C$ is a $k$-map satisfying (3) and

(4) $\sum(a b)_{\psi} \otimes c^{\psi}=\sum a_{\phi} b_{\psi} \otimes c^{\psi \phi}$;

(5) $\sum a_{\psi} 1_{\phi} \otimes c^{\psi}{ }_{1}^{\phi} \otimes c^{\psi}{ }_{2}=\sum a_{\psi \phi} \otimes c_{1}{ }^{\psi} \otimes c_{2}{ }^{\phi}$.

We adopt the notation $\psi(c \otimes a)=\sum a_{\psi} \otimes c^{\psi}$. If the map $\psi$ occurs more than once in the same expression, then we use notations $\phi, \delta, \bar{\psi}, \cdots$.

Remark 2. A partial entwining structure $(A, C, \psi)$ is an entwining structure if and only if $\sum 1_{\psi} \otimes c^{\psi}=1 \otimes c$.

Example 3. Suppose $H$ is a Hopf algebra with an idempotent $e$ verifying $\Delta(e)(e \otimes 1)=e \otimes e$ and $\varepsilon(e)=1$. Taking $A:=H, C:=H$ and $\psi: C \otimes A \rightarrow$ $A \otimes C, c \otimes a \mapsto a \otimes c e$, it is easy to prove that $(A, C, \psi)$ is a partial entwining structure.

Definition 4. Let $(A, C, \psi)$ is a right-right partial entwining structure. A partial entwined module $M$ over $(A, C, \psi)$ is a right $A$-module endowed a $k$-map $\rho$ : $M \rightarrow M \otimes C, m \mapsto \sum m_{[0]} \otimes m_{[1]}$ such that the following are satisfied for any $m \in M, a \in A$ :

(6) $\sum m_{[0][0]} \otimes m_{[0][1]} \otimes m_{[1]}=\sum m_{[0]} \cdot 1_{\phi \psi} \otimes m_{[1] 1} \psi \otimes m_{[1] 2} \phi ;$

(7) $\sum \varepsilon\left(m_{[1]}\right) m_{[0]}=m$;

(8) $\sum(m \cdot a)_{[0]} \otimes(m \cdot a)_{[1]}=\sum m_{[0]} \cdot a_{\psi} \otimes m_{[1]} \psi$.

A morphism between two partial entwined modules $M$ and $N$ is a right $A$-linear map $f: M \rightarrow N$ such that $\sum f(m)_{[0]} \otimes f(m)_{[1]}=\sum f\left(m_{[0]}\right) \otimes m_{[1]} . M_{A}^{C}(\psi)$ denotes the category of partial entwined modules over $(A, C, \psi)$.

Example 5. Let $(A, C, \psi)$ is a right-right partial entwining structure and $M$ a right $A$-module, and let $p=\left(\phi_{M} \otimes i d_{C}\right) \circ\left(i d_{M} \otimes \psi\right) \circ\left(i d_{M \otimes C} \otimes u_{A}\right)$ and $\mathcal{D}=$ Imp, where $\phi_{M}$ denotes the right action of $M$ and $u_{A}$ the unit of the algebra $A$. Then it is easy to verify that $p^{2}=p$ and we claim that $\mathcal{D}$ is a partial entwined module with the action given by $p(m \otimes c) \cdot a=\sum p\left(m \cdot a_{\psi} \otimes c^{\psi}\right)$ and the coaction given by $\sum p(m \otimes c)_{[0]} \otimes p(m \otimes c)_{[1]}=\sum p\left(m \otimes c_{1}\right) \cdot 1_{\psi} \otimes c_{2}{ }^{\psi}$ for any $a \in A, m \in M, c \in C$. 
Firstly we show that the action is well-defined. In fact, for any $a, b \in A, m \in$ $M, c \in C$,

$$
\begin{aligned}
p((m \otimes c-p(m \otimes c)) \cdot a) & =\sum p\left(m \cdot a_{\phi} \otimes c^{\phi}-p\left(m \cdot a_{\psi} \otimes c^{\psi}\right)\right) \\
& =p\left(m \cdot a_{\phi} \otimes c^{\phi}\right)-p^{2}\left(m \cdot a_{\phi} \otimes c^{\phi}\right)=0 ; \\
\sum p(m \otimes c) \cdot a b & =\sum p\left(m \cdot(a b)_{\psi} \otimes c^{\psi}\right) \\
& =\sum p\left(m \cdot a_{\psi} b_{\phi} \otimes c^{\psi \phi}\right)=\sum(p(m \otimes c) \cdot a) \cdot b .
\end{aligned}
$$

Obviously $p(m \otimes c) \cdot 1=p(m \otimes c)$.

Next we show that the coaction is well-defined. Indeed, for $m \in M, c \in C$,

$$
\begin{aligned}
& \sum \rho(p(m \otimes c)) \\
= & \sum p\left(m \otimes c_{1}\right) \cdot 1^{\phi} \otimes c_{2}{ }^{\phi}=\sum p\left(m \cdot 1_{\phi \psi} \otimes c_{1}{ }^{\psi}\right) \otimes c_{2}{ }^{\phi} \\
= & \sum m \cdot 1_{\phi \psi} 1_{\eta} \otimes c_{1}{ }^{\psi \eta} \otimes c_{2}{ }^{\phi}=\sum m \cdot 1_{\phi \eta} \otimes c_{1}{ }^{\eta} \otimes c_{2}{ }^{\phi}=\rho(m \otimes c) .
\end{aligned}
$$

Finally we show that the compatibility condition are satisfied. In fact, for any $a \in A, m \in M, c \in C$,

$$
\begin{aligned}
& \sum \varepsilon\left(p(m \otimes c)_{[1]}\right) p(m \otimes c)_{[0]} \\
= & \sum \varepsilon\left(c_{2}^{\psi}\right) p\left(m \otimes c_{1}\right) \cdot 1_{\psi}=p(m \otimes c) ; \\
& \sum p(m \otimes c)_{[0]} \cdot 1_{\phi \psi} \otimes p(m \otimes c)_{[1] 1}{ }^{\psi} \otimes p(m \otimes c)_{[1] 2}{ }^{\phi} \\
= & \sum p\left(m \otimes c_{1}\right) \cdot 1_{\delta} 1_{\psi \phi} \otimes c_{2}{ }^{\delta}{ }_{1}^{\phi} \otimes c_{2}{ }_{2}{ }^{\psi} \\
= & \sum p\left(m \otimes c_{1}\right) \cdot 1_{\delta} 1_{\psi} 1_{\phi} \otimes c_{2}{ }^{\delta \psi}{ }_{1}^{\phi} \otimes c_{2}{ }^{\delta \psi}{ }_{2} \\
= & \sum p\left(m \otimes c_{1}\right) \cdot 1_{\delta} 1_{\psi} \otimes c_{2}{ }^{\delta}{ }_{1}^{\psi} \otimes c_{2}{ }_{2} \\
= & p(m \otimes c)_{[0][0]} \otimes p(m \otimes c)_{[0][1]} \otimes p(m \otimes c)_{[1]} ; \\
& \sum(p(m \otimes c) \cdot a)_{[0]} \otimes(p(m \otimes c) \cdot a)_{[1]}=\sum p\left(m \cdot a_{\psi} 1_{\phi \delta} \otimes c^{\psi}{ }_{1}^{\delta}\right) \otimes c^{\psi}{ }_{2}^{\phi} \\
= & \sum p\left(m \cdot a_{\psi} 1_{\phi} 1_{\delta} \otimes c^{\psi \phi}{ }_{1}{ }^{\delta}\right) \otimes c^{\psi \phi}{ }_{2}=\sum p\left(m \cdot a_{\psi} 1_{\delta} \otimes c^{\psi}{ }_{1}{ }^{\delta}\right) \otimes c^{\psi}{ }_{2} \\
= & \sum p\left(m \cdot a_{\psi \delta} \otimes c_{1}{ }^{\delta}\right) \otimes c_{2}{ }^{\psi}=\sum p(m \otimes c)_{[0]} \cdot a_{\psi} \otimes p(m \otimes c)_{[0]}{ }^{\psi} .
\end{aligned}
$$

Theorem 6. The forgetful functor $F: M_{A}^{C}(\psi) \rightarrow M_{A}$ has a right adjoint $G: M_{A} \rightarrow M_{A}^{C}(\psi)$.

Proof. By Example 5 we know that $\mathcal{D}$ is an object in $M_{A}^{C}(\psi)$ for any $M \in$ $M_{A}$. Thus we define a functor $G: M_{A} \rightarrow M_{A}^{C}(\psi)$ as follows: for any $M \in$ $M_{A}, G(M)=\mathcal{D}$; for any $f: M \rightarrow N \in M_{A}, G(f)=f \otimes i d_{C}$. 
Firstly we prove that $G(f)$ is well-defined for any $f: M \rightarrow N \in M_{A}$. In fact, for any $m \in M, c \in C, G(f)(p(m \otimes c))=\sum f\left(m \cdot 1_{\phi} \otimes c^{\phi}\right)=\sum f(m) \cdot 1_{\phi} \otimes c^{\phi}=$ $G(f)(m \otimes c)$.

Secondly we prove that $G(f)$ is a morphism in $M_{A}^{C}(\psi)$. Indeed, for any $m \in$ $M, c \in C, a \in A$,

$$
\begin{aligned}
& G(f)(p(m \otimes c) \cdot a) \\
= & \sum f\left(m \cdot a_{\phi}\right) \otimes c^{\phi}=\sum f(m) \cdot a_{\phi} \otimes c^{\phi}=G(f)(p(m \otimes c)) \cdot a ; \\
& \left(\left(G(f) \otimes i d_{C}\right) \circ \rho_{\mathcal{D}}\right)(p(m \otimes c))=\sum f\left(m \cdot 1_{\phi \delta} 1_{\eta} \otimes c_{1}^{\delta \eta}\right) \otimes c_{2}{ }^{\psi} \\
= & \sum f(m) \cdot 1_{\phi} 1_{\delta} \otimes c^{\phi_{1}}{ }^{\delta} \otimes c^{\phi}{ }_{2} \\
= & \sum f(m) \cdot 1_{\phi} 1_{\eta \delta} \otimes c^{\phi^{\delta}}{ }_{1}^{\delta} \otimes c^{\phi_{2}}{ }^{\eta}=\left(\rho_{p(N \otimes C)} \circ G(f)\right)(p(m \otimes c)) .
\end{aligned}
$$

Thirdly we define the unit $\kappa$ of the adjoint. For any $M \in M_{A}^{C}(\psi), \kappa_{M}: M \rightarrow$ $\mathcal{D}, m \mapsto \sum p\left(m_{[0]} \otimes m_{[1]}\right)$. Then $\kappa_{M} \in M_{A}^{C}(\psi)$. In fact, for any $m \in M, a \in A$,

$$
\begin{aligned}
\kappa_{M}(m \cdot a) & =\sum p\left((m \cdot a)_{[0]} \otimes(m \cdot a)_{[1]}\right) \\
& =\sum p\left(m_{[0]} \cdot a_{\psi} \otimes m_{[1]}^{\psi}\right) \\
& =p\left(m_{[0]} \otimes m_{[1]}\right) \cdot a=\kappa_{M}(m) \cdot a ;\left(\kappa_{M} \otimes i d\right) \circ \rho_{M}(m) \\
& =\sum p\left(m_{[0][0]} \otimes m_{[0][1]}\right) \otimes m_{[1]} \\
& =\sum p\left(m_{[0]} \cdot 1_{\phi \psi} \otimes m_{[1] 1}^{\psi}\right) \otimes m_{[1] 2}{ }^{\phi} \\
& =\sum p\left(m_{[0]} \otimes m_{[1]}\right) \cdot 1_{\phi} \otimes m_{[1] 2}{ }^{\phi}=\rho_{\mathcal{D}}\left(\kappa_{M}(m)\right) .
\end{aligned}
$$

Next we define the counit $\iota$ of the adjoint. For any $M \in M_{A}, \iota_{M}: \mathcal{D} \rightarrow$ $M, p(m \otimes c) \mapsto \varepsilon(c) m$. Hence we have to prove that $\iota_{M}$ is well-defined. Indeed, for any $m \in M, c \in C, \iota_{M}\left(p(m \otimes c)=\sum \varepsilon\left(c^{\psi}\right)\left(m \cdot 1_{\psi}\right)=\varepsilon(c) m=\iota_{M}(m \otimes c)\right.$. It is very easy to verify $\iota$ is right $A$-linear.

Finally we will prove $G\left(\iota_{M}\right) \circ \kappa_{G(M)}=i d_{G(M)}$ and $\iota_{F(N)} \circ F\left(\kappa_{G(N)}\right)=$ $i d_{F(N)}$ for any $M \in M_{A}, N \in M_{A}^{C}(\psi)$. In fact, for any $m \in M, c \in C, n \in$ $N, G\left(\iota_{M}\right) \circ \kappa_{G(M)}(p(m \otimes c))=\sum \varepsilon\left(c_{2}{ }^{\phi \psi}\right) p\left(m \otimes c_{1}\right) \cdot 1_{\phi} 1_{\psi}=p(m \otimes c)$ and $\iota_{F(N)} \circ F\left(\kappa_{G(N)}\right)(m)=\sum \varepsilon\left(m_{[1]}^{\phi}\right) m_{[0]} \cdot 1_{\phi}=m$.

Therefore we complete the proof.

It is well known that the classical Maschke's theorem has been generalized in many forms such as Maschke-type theorems for Doi-Hopf modules, entwined modules[1],[2] and weak entwined modules[9]. Now we will give a Maschke-type theorem for a partial entwined module. 
Definition 7. Let $(A, C, \psi)$ be a partial entwining structure and $\sigma: C \rightarrow$ $C^{*} \otimes A, c \mapsto \sum c^{(1)} \otimes c^{(2)}$ a $k$-module map. We call the map $\sigma$ a normalized integral map in $(A, C, \psi)$ if the following conditions are satisfied for any $c, d \in C$ and $a \in A$ :

(12) $\sum d^{\psi(1)}\left(c^{\phi}\right) a_{\psi \phi} d^{\psi(2)}=\sum d^{(1)}(c) d^{(2)} a$;

(13) $\sum d^{\delta}{ }_{1}^{\phi(1)}\left(c^{\psi}\right)\left(1_{\delta} 1_{\phi}\right)_{\psi} d^{\delta}{ }_{1}{ }^{\phi(2)} \otimes d^{\delta}{ }_{2}=\sum d^{(1)}\left(c_{2}{ }^{\phi}\right) 1_{\phi \psi} d^{(2)}{ }_{\delta} \otimes c_{1} \psi \delta ;$

(14) $\sum 1_{\phi \psi} c_{2}{ }^{\phi(1)}\left(c_{1}{ }^{\psi}\right) c_{2}{ }^{\phi(2)}=\sum \varepsilon(c) 1$.

Lemma 8. Let $(A, C, \psi)$ be a partial entwining structure with a normalized integral map $\sigma, M, N$ in $M_{A}^{C}(\psi)$ and $f$ a morphism in $M_{A}$. Then the map $\bar{f}$ : $M \rightarrow N, m \mapsto \sum m_{[1]}^{(1)}\left(f\left(m_{[0]}\right)_{[1]}\right) f\left(m_{[0]}\right)_{[0]} \cdot m_{[1]}^{(2)}$ is a morphism in $M_{A}^{C}(\psi)$.

Proof. First we show that $\bar{f}$ is right $A$-linear. In fact, for any $m \in M$ and $a \in A$,

$$
\begin{aligned}
& \bar{f}(m \cdot a)=\sum(m \cdot a)_{[1]}{ }^{(1)}\left(f\left((m \cdot a)_{[0]}\right)_{[1]}\right) f\left((m \cdot a)_{[0]}\right)_{[0]} \cdot(m \cdot a)_{[1]}{ }^{(2)} \\
& =\sum m_{[1]}^{\psi(1)} f\left(m_{[0]} \cdot a_{\psi}\right)_{[1]} f\left(m_{[0]} \cdot a_{\psi}\right)_{[0]} \cdot m_{[1]}^{\psi(2)} \\
& =\sum m_{[1]} \psi(1)\left(f\left(m_{[0]}\right) \cdot a_{\psi}\right)_{[1]}\left(f\left(m_{[0]}\right) \cdot a_{\psi}\right)_{[0]} \cdot m_{[1]} \psi(2) \\
& =\sum m_{[1]}^{\psi(1)}\left(f\left(m_{[0]}\right)_{[1]}^{\phi}\right) f\left(m_{[0]}\right)_{[0]} \cdot a_{\psi \phi} m_{[1]} \psi(2) \\
& =\sum m_{[1]}^{(1)}\left(f\left(m_{[0]}\right)_{[1]}\right) f\left(m_{[0]}\right)_{[0]} \cdot m_{[1]}^{(2)} a=\bar{f}(m) \cdot a,
\end{aligned}
$$

where we get the second and the fourth equations from the definition of $M_{A}^{C}(\psi)$, and we obtain the third equation because $f$ is right $A$-linear.

Next we prove that $\bar{f}$ is right $C$-colinear. Indeed,for any $m \in M$ we also have,

$$
\begin{aligned}
& \sum f(m)_{[0]} \otimes f(m)_{[1]} \\
& =\sum m_{[1]}^{(1)}\left(f\left(m_{[0]}\right)_{[1]}\right)\left(f\left(m_{[0]}\right)_{[0]} \cdot m_{[1]}^{(2)}\right)_{[0]} \otimes\left(f\left(m_{[0]}\right)_{[0]} \cdot m_{[1]}^{(2)}\right)_{[1]} \\
& =\sum m_{[1]}^{(1)}\left(f\left(m_{[0]}\right)_{[1] 2}{ }^{\phi}\right) f\left(m_{[0]}\right)_{[0]} \cdot 1_{\phi \psi} m_{[1]}{ }^{(2)}{ }_{\delta} \otimes f\left(m_{[0]}\right)_{[1] 1} \psi \delta \\
& =\sum m_{[1]}^{\delta}{ }_{1}^{\phi(1)}\left(f\left(m_{[0]}\right)_{[1]}{ }^{\psi}\right) f\left(m_{[0]}\right)_{[0]} \cdot\left(1_{\delta} 1_{\phi}\right)_{\psi} m_{[1]}{ }_{1}^{\phi}{ }^{\phi(2)} \otimes m_{[1]}{ }^{\delta}{ }_{2} \\
& =\sum m_{[1] 1}{ }^{\phi(1)}\left(f\left(m_{[0]}\right) \cdot 1_{\delta \phi}\right)_{[1]}\left(f\left(m_{[0]}\right) \cdot 1_{\delta \phi}\right)_{[0]} \cdot m_{[1] 1} \phi(2) \otimes m_{[1] 2} \delta \\
& =\sum m_{[0][1]}^{(1)}\left(f\left(m_{[0][0]}\right)\right)_{[1]}\left(f\left(m_{[0][0]}\right)\right)_{[0]} \cdot m_{[0][1]}^{(2)} \otimes m_{[1]} \\
& =\sum f\left(m_{[0]}\right) \otimes m_{[1]}
\end{aligned}
$$

where we get the fourth equation by the fact that $N$ is an object in $M_{A}^{C}(\psi)$. 
Theorem 9. If there exists a normalized integral map in $(A, C, \psi)$, then a morphism in $M_{A}^{C}(\psi)$ has a section(resp. retraction) in $M_{A}$ has a section(resp. retraction) in $M_{A}^{C}(\psi)$.

Proof. Let $M, N \in M_{A}^{C}(\psi)$, and assume that $f: M \rightarrow N \in M_{A}^{C}(\psi)$ has a section $g: N \rightarrow M \in M_{A}$. Let $\bar{g}$ as mentioned in lemma 8, then for any $n \in N$,

$$
\begin{aligned}
& (f \circ \bar{g})(n)=\sum n_{[1]}^{(1)}\left(g\left(n_{[0]}\right)_{[1]}\right) f\left(g\left(n_{[0]}\right)_{[0]} \cdot n_{[1]}^{(2)}\right) \\
= & \sum n_{[1]}^{(1)} f g\left(n_{[0]}\right)_{[1]} f g\left(n_{[0]}\right)_{[0]} \cdot n_{[1]}^{(2)} \\
= & \sum n_{[1] 2}^{\phi(1)}\left(n_{[1] 1}^{\psi}\right) n_{[0]} \cdot 1_{\phi \psi} n_{[1] 2}{ }^{(2)}=n .
\end{aligned}
$$

where we get the second equation from the fact that $f \in M_{A}^{C}(\psi)$, and we obtain the last equation by Definition 7.

\section{REFERENCES}

1. Tomasz Brzezinski, Frobenius Properties and Maschke-type Theormes for Entwined Modules, Proc. American Math. Soc., 128(8) (1999), 2261-2279.

2. S. Caenepeel, G. Militaru and Zhu Shenlin, A Maschke Type Theorem for Doi-Hopf Modules and Applications, J. Algebra, 187 (1997), 388-412.

3. S.Caenepeel and K. Janssen, Partial Entwining Structures, Preprint.

4. S. Caenepee and, E. De Groot, Corings Applied to Partial Galois Theory, Proceedings of the International Coference on Mathematics and Applications, ICMA, 2004, S. L. Kalla and M. M. Chawla (eds.), Kuwait University, Kuwait, 2005, pp. 117-134.

5. M. Dokuchaev, R. Exel and P. Piccione, Partial Representations and Partial Group Algebras, J. Algebra, 226(1) (2000), 251-268.

6. M. Dokuchaev and R. Exel, Associativity of Crossed Products by Partial Actions, Enveloping Actions and Partial Representations, Trans. Amer. Math. Soc., 357 (2005), 1931-1952.

7. M. Dokuchaev and N. Zhukavets, On Finite Degree Partial Representations of Groups, J. Algebra, 274 (2004), 309-334.

8. R. Exel, Twisted Partial Actions: A Classification of Regular $C^{*}$-algebraic Bundels, Proc. Londom Math. Soc., 74 (1997), 417-443.

9. Ling Jia and Fang Li, Frobenius Properties and Maschke-type Theormes for Weak Entwined Modules, Acta Mathematica Sinica (Chinese), 50(1) (2007), 105-116.

Ling Jia

Department of Mathematics and Information,

Ludong University,

Yantai, Shandong 264025,

P. R. China

E-mail: jialing471@126.com 